\title{
An Algebraic Approach to the Harmonic Oscillator Plus an Inverse Square Potential in Three Dimensions
}

\author{
Shi-Hai Dong ${ }^{1}$ and M. Lozada-Cassou ${ }^{2}$ \\ Programa de Ingeniería Molecular, Instituto Mexicano del Petróleo \\ Lázaro Cárdenas 152, 07730 México, D. F., México
}

\begin{abstract}
The eigenfunctions and eigenvalues of the three-dimensional Schrödinger equation with a harmonic oscillator plus an inverse square interaction are obtained. A realization of the ladder operators for the wave functions is studied. It is found that these operators satisfy the commutation relations of an $\mathrm{SU}(1,1)$ group. The closed analytical expressions for the matrix elements of different functions $\rho$ and $\rho \frac{d}{d \rho}$ with $\rho=r^{2}$ are evaluated. Another hidden symmetry explores the relations between the eigenvalues and eigenfunctions for substituting $r \rightarrow i r$.

PACS number(s): 03. 65. Fd, 03. 65.Ge and 02. 20.Qs.
\end{abstract}

Key words: Inverse Square Interaction, Ladder Operators, SU $(1,1)$ Group, Matrix Elements

\section{INTRODUCTION}

It is well known that the algebraic method has been the subject of the interest in the wide variety of fields of physics. Systems displaying a dynamical symmetry can be treated by algebraic techniques $[1,2,3,4]$. With the factorization method $[5,6,7]$, we have established the ladder operators of a quantum system with some important potentials such as the Morse potential, the Pöschl-Teller one, the pseudoharmonic one, the infinitely square-well one and other quantum systems $[8,9,10,11,12,11,12,13,14,15,16,17,18]$. From those ladder operators we can finally constitute a suitable algebra and simultaneously obtain the matrix elements for some related functions. It should be addressed that our approach is different from the traditional one, where an auxiliary variable was introduced [19], namely, we can construct the ladder operators only from the physical variable without introducing any auxiliary variable. Recently, the quantum system for the one-dimensional harmonic oscillator plus an inverse square interaction has been studied [20]. To our knowledge, however, the hidden symmetry of this quantum system is a gap to be filled in, which is the main purpose of the present work.

The Eigenvalues and Eigenfunctions: Now, we are studying the exact solutions, which are necessary to be used to construct the ladder operators and constitute a suitable algebra. Consider the Schrödinger equation with a potential $V(r)$ that depends only on the distance $r$ from the origin:

$$
-\frac{\hbar^{2}}{2 \mu} \nabla^{2} \psi(r, \theta, \varphi)+V(r) \psi(r, \theta, \varphi)=E \psi(r, \theta, \varphi),
$$

where $\mu$ is the mass of the particle. In the present work, the potential $V(r)$ is taken as the harmonic potential plus an inverse square interaction,

$$
V(r)=\frac{1}{2} \mu \omega^{2} r^{2}+\frac{\hbar^{2}}{2 \mu} \frac{\alpha}{r^{2}}
$$

where, the $\omega$ is the frequency and $\alpha$ is the strength of the external field.

For simplicity, the natural units $\hbar=\mu=\omega=1$ are employed throughout this paper if not explicitly stated otherwise. Owing to the symmetry of the potential, we take the wave functions with the form

\footnotetext{
${ }^{1}$ E-mail address: dongsh2@yahoo.com

${ }^{2}$ E-mail address: marcelo@www.imp.mx
} 


$$
\psi(r, \theta, \varphi)=R(r) Y_{l m}(\theta, \varphi)
$$

where, the radial wave function $R(r)$ satisfies the following equation

$$
\frac{d^{2} R(r)}{d r^{2}}+\frac{2}{r} \frac{d R(r)}{d r}+\left[2(E-V(r))-\frac{l(l+1)}{r^{2}}\right] R(r)=0 .
$$

Defining the new variable $\rho=r^{2}$, equation (4) can be re-arranged as

$$
\frac{d^{2}}{d \rho^{2}} R(\rho)+\frac{1}{\rho} \frac{d}{d \rho} R(\rho)-\left(\frac{1}{4}+\frac{\alpha+l(l+1)}{4 \rho^{2}}-\frac{E}{2 \rho}\right) R(\rho)=0 .
$$

From the behaviors of the wave functions at the origin and at infinity, we can take the following ansatz for the wave functions

$$
R(\rho)=\rho^{s} e^{-\frac{\rho}{2}} F(\rho)
$$

with

$$
s=\frac{-1 / 2+\sqrt{\alpha+(l+1 / 2)^{2}}}{2},
$$

where, another solution $s=\left(-1 / 2-\sqrt{\alpha+(l+1 / 2)^{2}}\right) / 2$ is not an acceptable one in physics. Substitution of Eq. (6) into Eq. (5) allows us to obtain

$$
\rho \frac{d^{2}}{d \rho^{2}} F(\rho)+(2 s+3 / 2-\rho) \frac{d}{d \rho} F(\rho)+(E / 2-s-3 / 4) F(\rho)=0,
$$

whose solution is nothing but the confluent hypergeometric solutions ${ }_{1} F_{1}(s-E / 2+3 / 4,2 s+3 / 2 ; \rho)$. One can finally obtain the eigenfunctions as

$$
R(\rho)=N \rho^{s} e^{-\frac{\rho}{2}} F_{1}(s-E / 2+3 / 4,2 s+3 / 2 ; \rho),
$$

with a normalized factor $N$ to be determined below:

From consideration of the finiteness of the solutions, it is shown from Eq. (7) that the general quantum condition is

$$
s-E / 2+3 / 4=-n, \quad n=0,1,2, \ldots,
$$

from which we have

$$
E_{n}=2(n+s+3 / 4)=3 / 2+2 n+2 s,
$$

which implies that the energy level is equidistant.

Recall that when $n=E / 2-s-3 / 4$ is a non-negative integer, the confluent hypergeometric functions can be expressed by the associated Laguerre polynomials [21]:

$$
L_{n}^{\alpha}(x)=\frac{\Gamma(\alpha+n+1)}{n ! \Gamma(\alpha+1)}{ }_{1} F_{1}(-n, \alpha+1 ; x),
$$

from which, together with the following important formula [21]: 


$$
\int_{0}^{\infty} x^{\alpha} e^{-x} L_{n}^{\alpha}(x) L_{m}^{\alpha}(x) d x=\frac{\Gamma(n+\alpha+1)}{n !} \delta_{n m}
$$

we can finally obtain the normalized radial wave functions

$$
R(\rho)=N_{n} \rho^{s} e^{-\frac{\rho}{2}} L_{n}^{2 s+1 / 2}(\rho),
$$

with

$$
N_{n}=\sqrt{\frac{2 n !}{(n+2 s+1 / 2) !}} .
$$

The construction of the ladder operators: We address the problem of finding the creation and annihilation operators for the radial wave functions (12) with the factorization method. [8-18], the ladder operators can be constructed directly from the wave functions without introducing any auxiliary variable, namely, we intend to find differential operators $\hat{L}_{ \pm}$with the following property

$$
\hat{L}_{ \pm} R_{n}(\rho)=l_{ \pm} R_{n \pm 1}(\rho) \text {. }
$$

Specifically, we look for operators of the form

$$
\hat{L}_{ \pm}=A_{ \pm}(\rho) \frac{d}{d \rho}+B_{ \pm}(\rho),
$$

where we stress that these operators only depend on the physical variable $\rho$.

To this end we start by establishing the action of the differential operator $\frac{d}{d \rho}$ on the wave functions (12)

$$
\frac{d}{d \rho} R_{n}(\rho)=\left(\frac{s}{\rho}-\frac{1}{2}\right) R_{n}(\rho)+N_{n} \rho^{s} e^{-\frac{\rho}{2}} \frac{d}{d \rho} L_{n}^{2 s+1 / 2}(\rho)
$$

One possible relation for the first derivative of the associated Laguerre functions is given in [21]

$$
x \frac{d}{d x} L_{n}^{\alpha}(x)=n L_{n}^{\alpha}(x)-(n+\alpha) L_{n-1}^{\alpha}(x) .
$$

The substitution of this expression into (16) enables us to obtain the following relation

$$
\left[\frac{d}{d \rho}-\frac{n+s}{\rho}+\frac{1}{2}\right] R_{n}(\rho)=-\frac{n+2 s+1 / 2}{\rho} \frac{N_{n}}{N_{n-1}} R_{n-1}(\rho) .
$$

Making use of Eq. (13) and introducing the number operator $\$$ hat $\{n\} \$$ with the property

$$
n R_{n}(\rho)=n R_{n}(\rho)
$$

we can define the following operator

$$
\hat{L}_{-}=-\rho \frac{d}{d \rho}+s+\hat{n}-\frac{\rho}{2},
$$

with the following effect over the wave functions

$$
\hat{L}_{-} R_{n}(\rho)=l_{-} R_{n-1}(\rho),
$$

where,

$$
l_{-}=\sqrt{n(n+2 s+1 / 2)} \text {. }
$$


As we can see, this operator annihilates the ground state $R_{0}(\rho)$, as expected from a step-down operator.

We now proceed to find the corresponding creation operator. Before proceeding to do so, we should make use of another relation between the associated Laguerre functions [21].

$$
x \frac{d}{d x} L_{n}^{\alpha}(x)=(n+1) L_{n+1}^{\alpha}(x)-(n+\alpha+1-x) L_{n}^{\alpha}(x)
$$

Substitution of this expression into Eq. (16) admits us to obtain

$$
\left[\frac{d}{d \rho}+\frac{n+s+3 / 2}{\rho}-\frac{1}{2}\right] R_{n}(\rho)=\frac{n+1}{\rho} \frac{N_{n}}{N_{n+1}} R_{n+1}(\rho) .
$$

Using Eq. (13) again, we can define the following operator

$$
\hat{L}_{+}=\rho \frac{d}{d \rho}+s+\hat{n}+1-\frac{\rho}{2},
$$

satisfying the equation

$$
\hat{L}_{+} R_{n}(\rho)=l_{+} R_{n+1}(\rho)
$$

with

$$
l_{+}=\sqrt{(n+1)(n+2 s+3 / 2)} .
$$

Therefore it is shown that the wave functions can be directly obtained from the creation operator $\hat{L}_{+}$acting on the ground state $R_{0}(\rho)$, namely,

$$
R_{n}(\rho)=N_{n} \hat{L}_{+}^{n} R_{0}(\rho)
$$

with

$$
N_{n}=\sqrt{\frac{(2 s+1 / 2) !}{n !(n+2 s+1 / 2) !}}, \quad R_{0}(\rho)=\sqrt{\frac{2}{(2 s+1 / 2) !}} \rho^{s} e^{-\frac{\rho}{2}}
$$

We now study the algebra associated to the operators $\hat{L}_{+}$and $\hat{L}_{-}$. Based on the results (21-22) and (26-27) we can calculate the commutator $\left[\hat{L}_{-}, \hat{L}_{+}\right]$:

$$
\left[\hat{L}_{-}, \hat{L}_{+}\right] R_{n}(\rho)=2 l_{0} R_{n}(\rho)
$$

where we have introduced the eigenvalue

$$
l_{0}=\left(n+s+\frac{3}{4}\right)
$$

We can thus define the operator

$$
\hat{l}_{0}=\left(\hat{n}+s+\frac{3}{4}\right)
$$

The operators $\hat{L}_{ \pm}$and $\hat{L}_{0}$ thus satisfy the commutation relations 


$$
\left[\hat{L}_{-}, \hat{L}_{+}\right]=2 \hat{L}_{0}, \quad\left[\hat{L}_{0}, \hat{L}_{-}\right]=-\hat{L}_{-}, \quad\left[\hat{L}_{0}, \hat{L}_{+}\right]=\hat{L}_{+},
$$

which correspond to an $\mathrm{SU}(1,1)$ algebra for the radial wave functions. The Casimir operator can be also expressed as

$$
\hat{C} R_{n}(\rho)=\left[\hat{L}_{0}\left(\hat{L}_{0}-1\right)-\hat{L}_{+} \hat{L}_{-}\right] R_{n}(\rho)=\left(s^{2}+s / 2-3 / 16\right) R_{n}(\rho) .
$$

The hamiltonian $\hat{H}$ can be expressed as:

$$
\hat{H}|n\rangle=2 \hat{L}_{0}|n\rangle=(2 n+2 s+3 / 2)|n\rangle .
$$

It is shown that there are four series of irreducible unitary representations for the $\mathrm{SU}(1,1)$ algebra except for the identity representation [22]. They are the representation $D^{ \pm}(j)$ with a spectrum bounded below and above, respectively; the supplementary series $D_{s}\left(Q, q_{0}\right)$ and the principle series $D_{p}\left(Q, q_{0}\right)$. Since the eigenvalues have the ground state, the representation of the dynamical group $\mathrm{SU}(1,1)$ belongs to $D^{+}(j)$ :

$$
\begin{aligned}
& I_{0}|j, v\rangle=v|j, v\rangle, \\
& I_{-}|j, v\rangle=[(v+j)(v-j-1)]^{1 / 2}|j, v-1\rangle, \\
& I_{+}|j, v-1\rangle=[(v+j)(v-j-1)]^{1 / 2}|j, v\rangle, \\
& v=-j+n, \quad n=0,1,2, \ldots, \quad j<0 .
\end{aligned}
$$

In comparison with Eqs. (21-22), (29-30) and (33-34) we have $j=-(s+3 / 4), \quad v=n+s+3 / 4$ and $R_{n}(\rho)=|j, v\rangle$.

On the other hand, the following expressions in terms of the creation and annihilation operators $\hat{L}_{ \pm}$and $\hat{L}_{0}$ can be obtained as

$$
\rho=2 \hat{L}_{0}-\hat{L}_{-}-\hat{L}_{+}
$$

and

$$
\rho \frac{d}{d \rho}=\frac{1}{2}\left(\hat{L}_{+}-\hat{L}_{-}-3 / 2\right)
$$

The matrix elements of these two functions can be analytically obtained in terms of Eqs. (21), (31) and (34) as

$$
\begin{aligned}
\left\langle R_{m}(\rho)|\rho| R_{n}(\rho)\right\rangle & \equiv \int_{0}^{\infty} R_{m}(r) r^{2} R_{n}(r) d r \\
& =(2 \mathrm{n}+2 \mathrm{~s}+3 / 2) \delta_{\mathrm{m}, \mathrm{n}} \\
& -\sqrt{n(n+2 s+1 / 2)} \delta_{\mathrm{m},(\mathrm{n}-1)} \\
& -\sqrt{(n+1)(n+2 s+3 / 2)} \delta_{\mathrm{m},(\mathrm{n}+1)}
\end{aligned}
$$

and 


$$
\begin{aligned}
\left\langle R_{m}(\rho)\left|\rho \frac{d}{d \rho}\right| R_{n}(\rho)\right\rangle & \equiv \int_{0}^{\infty} R_{m}(r) \frac{r}{2} \frac{d}{d r} R_{n}(r) d r \\
& =\frac{1}{2} \sqrt{(n+1)(n+2 s+3 / 2)} \delta_{\mathrm{m},(\mathrm{n}+1)} \\
& -\frac{1}{2} \sqrt{n(n+2 s+1 / 2)} \delta_{\mathrm{m},(\mathrm{n}-1)} \\
& -\frac{3}{4} \delta_{\mathrm{m}, \mathrm{n}}
\end{aligned}
$$

where, the integral range $r \in(0, \infty)$. It is shown that this is a very simple method to calculate the matrix elements from these ladder operators.

Before ending this section, it should be pointed out that there exists another hidden symmetry for this quantum system. As discussed in our previous work [23], it is found that the eigenfunctions (12) (the variable $\rho=r^{2}$ ) vanish not only as $r \rightarrow \infty$, bus also as $r \rightarrow i \infty$. The substitution $r \rightarrow i r$, also demonstrates intimate connections between the energies, that is to say, the eigenvalues $E$ of quantum system will change to $-E$ but keeping other parameters unchanged.

\section{CONCLUSION}

In this paper we have studied the eigenvalues and eigenfunctions for the one-dimensional harmonic oscillator plus an inverse square interaction and then established the creation and annihilation operators directly from the eigenfunctions (12) with the factorization method. We have derived a realization of dynamic group only in terms of the physical variable $\rho$, without introducing any auxiliary variable. It is shown that these operators satisfy an $\mathrm{SU}(1,1)$ dynamic group. The representation of the bound states of this quantum system is described by the representation $D^{+}(j)$ with a spectrum bound below. The matrix elements of the different functions $\rho$ and $\rho d / d \rho$ with $\rho=r^{2}$ are also analytically obtained from the ladder operators $\hat{L}_{ \pm}$ and $\hat{L}_{0}$. This method can be generalized to other wavefunctions and represents a simple and elegant approach to obtain these matrix elements.

\section{ACKNOWLEDGMENT}

This work is supported by CONACyT, Mexico.

\section{REFERENCES}

1. Arima, A. and F. Iachello, 1974. Interacting boson model of collective states I. The vibrational limit. Ann. Phys., 99: 253.

2. Perelomov, A.,1985.Generalized Coherent States and their Applications. New York, Springer.
3. Wybourne, R.G., 1974. Classical Groups for Physicists. New York, Wiley.

4. Cooper, I.L., 1993. An integrated approach to ladder and shift operators for the Morse oscillator, radial Coulomb and radial oscillator potentials. J. Phys. A: Math. Gen., 26: 1601.

5. Schrödinger, E., 1940. A method of determining quantum mechanics eigenvalues and eigenfunctions. Pro. R.Irish Acad. A 46 No. 9, 183.

6. Infeld, L. and T.E. Hull, 1951. The factorization method. Rev. Mod. Phys., 23: 21.

7. Haidinger, G., N. Bessis and G. Bessis, 1974. Closed-form expressions of matrix elements and eigenfunctions from Ladder-operator considerations. J. Math. Phys. 15: 716.

8. Dong, S.H., R. Lemus and A. Frank, 2002. Ladder operators for the Morse potential. Int. J. Quan. Chem., 86: 433.

9. Dong, S. H. and R. Lemus, 2002. Ladder operators for the modified Pöschl-Teller potential. Int. J. Quan. Chem., 86: 265.

10. Dong, S.H.2002. The SU(2) realization for the Morse potential and its coherent states.Can. J. Phys., 80: 129.

11. Dong, S.H.,2002. Algebraic approach to the Morse potential and its coherent states. Z. Phys. Chem., 216: 103.

12. Dong, S.H. and Z.Q. Ma, 2002. The hidden symmetry for a quantum system with an infinitely deep square-well potential. Am. J. Phys., 70: 520.

13. Dong, S.H. and Z.Q. Ma, 2002. Algebraic approach to the pseudoharmonic oscillator in 2D. Int. J. Mod. Phys., E 11: 155.

14. Dong, S. and S.H. Dong, 2002. An alternative approach to study the dynamical group for the modified Poschl-Teller potential. Czech. J. Phys., 52: 753.

15. Dong, S. and S.H. Dong, 2002. A realization of dynamic group for an electron in a uniform magnetic field. Int. J. Mod. Phys., E 11: 265.

16. Dong, S.H., 2003. The realization of dynamic group for the pseudoharmonic oscillator. Appl. Math. Lett., 16:199.

17. Dong, S.H., 2004. Realization of the dynamical group for the generalized Laguerre functions. 
Computers and Mathematics with applications 47: 1037.

18. Dong, S.H., G.H. Sun and Y. Tang, 2003. The hidden symmetry for a quantum system with a Poschl-Teller-like potential. Int. J. Mod. Phys., E 12: 809 .

19. Berrondo, M. and A. Palma, 1980. The algebraic approach to the Morse oscillator. J. Phys. A: Math. Gen. 13: 773.

20. Palma, G. and U. Raff, 2003. The one dimensional harmonic oscillator in presence of a dipole-like interaction. Am. J. Phys., 71: 247.
21. Gradshteyn, I.S. and I.M. Ryzhik, 1994. Tables of Integrals, Series and Products. $5^{\text {th }}$ Edn. Academic Press, New York.

22. Adams, B.G., J. Cizek and J. Paldus, 1987. Lie algebraic methods and their applications to simple quantum systems. In Advances in Quantum Chemistry Vol. 19, Academic Press, New York.

23. Child, M.S., S.H. Dong and X.G. Wang, 2000. Quantum states of a sextic potential: hidden symmetry and quantum monodromy. J. Phys. A: Math.Gen.,33:5653. 